\title{
Relationship between Retinal Nerve Fiber Layer Thickness and Aortic Distensibility in Peripheral Arterial Disease Patients
}

\author{
Deniz Kumova ${ }^{1}$, Zeynep Aktas $^{2}$, Azmi Eyiol ${ }^{3}$, Murat Hasanreisoglu ${ }^{4}$, Mustafa Cemri ${ }^{5}$
}

\begin{abstract}
Aim and objective: To evaluate the relationship between aortic distensibility (AD) and aortic stiffness B index (ASBI) with retinal nerve fiber layer (RNFL) thickness measured with HD-OCT in peripheral arterial disease (PAD) patients.

Materials and methods: Twenty-six PAD patients and 22 age-matched healthy control were enrolled. Subjects with PAD were classified into two groups. Patients with diabetes (DM) or hypertension (HT) comprised group I $(n=18)$ and without DM or HT comprised group II $(n=8)$. Color Doppler imaging was performed on all patients and PAD was diagnosed by using the ankle-brachial index (ABI). Retinal nerve fiber layer thickness values between control and PAD patients and correlations between RNFL thickness and aortic stiffness parameters (AD and ASBI) were evaluated.

Results: The inferior-nasal and inferior-temporal quadrant were the thickest in healthy subjects and the PAD group. Retinal nerve fiber layer thickness significantly decreased in superior-nasal, temporal, inferior-nasal quadrants in group I than healthy subjects $(p<0.001, p=0.005$, $p<0.001)$. Temporal and inferior-nasal quadrant thicknesses were statistically significantly thinner in group Il than controls $(p=0.02, p<0.001)$. The nasal RNFL quadrant was significantly thinner in group I than group II $(p=0.014)$. The correlation between RNFL thickness and aortic elasticity parameters in each group was not found to be significant.

Conclusion and clinical significance: Isolated PAD without DM or HT may lead to localized RNFL loss in temporal and inferior-nasal quadrants. Aortic elasticity parameters did not seem to be correlated with RNFL thickness in PAD.

Keywords: Aortic distensibility, Aortic stiffness, Peripheral arterial disease, Retinal nerve fiber layer.

Journal of Current Glaucoma Practice (2021): 10.5005/jp-journals-10078-1300
\end{abstract}

\section{INTRODUCTION}

Peripheral artery disease is a disease that develops due to atherosclerosis in which vessels other than cerebral and coronary arteries are involved. ${ }^{1,2}$ Atherosclerotic peripheral artery disease; it is a multifactorial disease that develops as a result of many risk factors that can be modified such as smoking, diabetes (DM), hypertension $(\mathrm{HT})$, and cannot be modified such as sex, age, and ethnicity. ${ }^{3-5}$

Atherosclerosis is a chronic, progressive disease in which many vascular beds such as the ocular circulation and optic nerve circulation are affected. ${ }^{6}$

Measurement methods of noninvasive atherosclerosis are limited. These are carotid echography or radiography used to measure intimal thickness, abdominal imaging modalities, and ankle-brachial index ( $\mathrm{ABI}$ ) used to detect calcification in the aorta. ${ }^{7-9}$ The $A B I$ is a simple, noninvasive, and objective test for detecting peripheral arterial disease (PAD). ${ }^{10,11}$

In general, an $\mathrm{ABI}$ value $<0.9$ is accepted as an indicator of atherosclerosis. ${ }^{11}$ Arterial stiffness expresses the degree of agerelated vascular degeneration and decreased vessel wall elasticity, which varies as a result of different pathologies. It is an important indicator of cardiovascular risk. ${ }^{12,13}$ Two important parameters used in the evaluation of regional aortic stiffness are the aortic distensibility (AD) and aortic stiffness B index (ASBI). ${ }^{14,15}$

Hayreh and Jonas demonstrated the association of chronic arterial HT and atherosclerosis with localized nerve fiber damage indicating optic nerve damage in experimental monkey models by detecting reduced visibility of the retinal nerve fiber layer (RNFL). ${ }^{16}$
${ }^{1}$ Department of Ophthalmology, Sultangazi Haseki Education Research Hospital, Istanbul, Turkey

${ }^{2}$ Department of Ophthalmology, Gazi University Medical Faculty, Ankara, Turkey

${ }^{3}$ Department of Cardiology, Beyhekim State Hospital, Konya, Turkey

${ }^{4}$ Department of Ophthalmology, Koç University, Faculty of Medicine, İstanbul, Turkey

${ }^{5}$ Department of Cardiology, Gazi University Medical School, Turkey

Corresponding Author: Deniz Kumova, Department of Ophthalmology, Sultangazi Haseki Education Research Hospital, Istanbul, Turkey, Phone: +905305295521, e-mail: denizkumova82@ gmail.com

How to cite this article: Kumova D, Aktas Z, Eyiol A, et al. Relationship between Retinal Nerve Fiber Layer Thickness and Aortic Distensibility in Peripheral Arterial Disease Patients. J Curr Glaucoma Pract 2021;15(2):86-90.

Source of support: Nil

Conflict of interest: None

We herein evaluated the relationship between the $A D$ and $A S B I$ with RNFL thickness in PAD patients, diagnosed according to $A B I$.

\section{Materials and Methods}

Twenty-six eyes of 26 patients with PAD and 22 eyes of 22 healthy controls were enrolled in our study. PAH patients were divided into two groups based on DM and HT status as follows: patients with 
DM or HT (group I, $n=18$ ) and patients without DM or HT (group II, $n=8$ ). An age-matched control group (group III) consisted of agematched healthy subjects. All participants were examined by the same cardiologist and ophthalmologist after they were informed and their written consent was obtained. After determining the best visual acuity with the Snellen chart of all participants, slit lamp examination, retinal, and optic nerve head examinations, and intraocular measurements (IOP) were performed. Intraocular measurements were measured three times with Goldmann applanation tonometry.

Patients with $20 / 20$ visual acuities, normal IOPs $(<21 \mathrm{~mm} \mathrm{Hg})$, and normal disc appearance were included in the study. However, patients with a known history of glaucoma, pigment dispersion or exfoliation, optic atrophy, any kind of retinopathy, neuroophthalmologic diseases, or media opacities preventing adequate posterior segment examination were excluded.

Ocular images of all participants were taken by the same experienced technician with HRT (Heidelberg Engineering $\mathrm{GmbH}$, software version 1.4.1.0) RNFL thicknesses in a total of six quadrants [superior temporal (ST), superior nasal (SN), temporal (T), nasal (N), inferior nasal (IN), inferior temporal (IT)] were measured and analyzed in both groups.

\section{Definition of Diabetes Mellitus and HT}

Hypertensive patients were determined by systolic blood pressure (BP) over than $140 \mathrm{~mm} \mathrm{Hg}$, diastolic BP over than $90 \mathrm{~mm} \mathrm{Hg}$, or using antihypertensive medication. Patients with secondary HT were excluded from the study. Diagnosis of DM was established with fasting glucose level above $100 \mathrm{mg} / \mathrm{dL}$ or postprandial glucose above $200 \mathrm{mg} / \mathrm{dL}$. Patients with previously diagnosed DM using systemic medications were also assumed as having DM after their medical records were controlled.

\section{Definition of PAD and ABI Assessment}

Ankle-brachial index measurements in all patients were measured with a standard Doppler USG (8 MHz KOVEN Hadeco Smartdop 20 Ultrasonic Blood Flow Detector, Japanese) from the ankle after resting for 5 minutes in the supine position.

The calculation of $A B I$ was performed by dividing the higher of the two systolic pressures (from dorsalis pedis and posterior tibial artery) measured at the ankle to the highest systolic pressure measured from the brachial artery in the upper extremity. Anklebrachial index $\leq 0.90$ was considered pathological. ${ }^{17}$

\section{Echocardiographic Assessment}

Conventional echocardiography was used to assess the elastic features of the aorta.

All patients included in the study were examined by the same cardiologist with $\mathrm{M}$ mode two-dimensional echocardiography in the left lateral decubitus position. Aortic diastolic and systolic inner diameters (AoD, AoS) of the ascending aorta $3 \mathrm{~cm}$ above the aortic valve in the parasternal long-axis were evaluated.

Aortic stiffness index and distensibility were calculated as follows:

Aortic distensibility $=2 \times($ AoS - AoD $) /(S P-D P) \times A o D\left(10^{-6}\right.$ $\mathrm{cm}^{2}$ dyn $^{-1}$ ),

Aortic stiffness index $=\ln [S P / D P) /(A o S-A o D) / A o D]$ (pure number),

In: natural logarithm

SP: systolic pressure

DP: diastolic pressure

\section{Statistical Analyses}

Statistical analysis was performed using SPSS. Retinal nerve fiber layer thickness between each group was compared by the Mann-Whitney $U$ test. Spearman's correlation test was performed to determine whether there was a correlation between the aortic stiffness parameter and RNFL thickness. $p$ value $<0.05$ was accepted statistically significant.

\section{Results}

The mean age in group I (PAD patients with DM or HT) was 62.3 \pm 10.0 years (range: $42-84$ years), and $63.7 \pm 14.8$ years in group II (PAD patients without DM or HT) (range: 32-77 years). In the healthy control group (group III), the mean age was $60.0 \pm 6.7$ (range: 59-62 years).

Mean ages, IOPs, and central corneal thickness (CCTs) of the patients were comparable in all groups (Table 1).

Retinal nerve fiber layer thicknesses in the healthy and PAD group are summarized in Table 2 . In group I, RNFL thicknesses in the temporal, superior-nasal, and inferior-nasal quadrants were significantly thinner than in group III. Nasal RNFL thicknesses were significantly different between group I and group II. However, other quadrant thicknesses were comparable (Table 3).

Aortic distensibility and stiffness index values of all groups are shown in Table 4. Aortic distensibility was significantly decreased in the PAD patients group (groups I and II) than group III, whereas a significant increase was found for the ASBI. However, in the current study, we did not find any correlation between RNFL thickness and aortic elasticity parameters in PAD patients (Table 5).

\section{Discussion}

$\mathrm{PAH}$ is indicative of generalized vascular atherosclerosis and often presents with atherosclerosis of lower extremity vessels. It is observed at a rate of $12-20 \%$ in the population over the age of 65 in the USA. ${ }^{18,19}$

However, claudication, which is the classic main symptom of the disease, is observed in only $10 \%$ of patients. ${ }^{20}$ Especially elderly patients are asymptomatic and the diagnosis of the disease can be easily missed in this group. ${ }^{21}$ The $A B I$ index is a useful and reliable noninvasive method used in the diagnosis of such patients.

Its specificity is $100 \%$ and its sensitivity is $95 \%$ in the detection of stenosis in the extremity arteries. ${ }^{1,2,17}$

Being highly reproducible and fast, it enables the diagnosis of the disease to be made at an early stage. Ankle-brachial index $<0.9$ makes the diagnosis of PAD. In this study, PAD was diagnosed in patients with an $\mathrm{ABI}<0.9$.

Systemic diseases such as DM can cause localized RNFL losses and these losses can be detected before the development of diabetic retinopathy. ${ }^{22-24}$

The severity of retinopathy, the age of the patient, and the presence of systemic HT are risk factors for RNFL losses in diabetes. ${ }^{25}$ Compromised in vascular reactivity and perfusion disorders cause RNFL thinning, especially in the upper quadrants. ${ }^{26,27}$

Other mechanisms of early onset of DM-associated RNFL loss were reported as enhanced apoptosis of neuroglial element and retrograde axonal transport impairment. ${ }^{28}$

In chronic HT, increasing vascular resistance over time can cause microvascular damage and cause atherosclerosis. Atherosclerosis 
Table 1: Mean age, intraocular pressure, and central corneal thickness values in all groups

\begin{tabular}{lllll}
\hline & Group I & Group II & Group III & \\
\cline { 2 - 4 } & $($ PAD with DM/HT) $n=18$ & $($ PAD without DM/HT) $n=8$ & (Controls) $n=22$ & 0.125 \\
\hline Age (years) & $62.3 \pm 10.0$ & $63.7 \pm 14.8$ & $60 \pm 6.7$ & $(59-62)$ \\
& $(42-84)$ & $(32-77)$ & $13.2 \pm 2.7$ & 0.235 \\
IOP $(\mathrm{mm} \mathrm{Hg})$ & $13.7 \pm 2.9$ & $13.8 \pm 1.0$ & $(10-16)$ & \\
& $(9-18)$ & $(9-17)$ & $549 \pm 14.01$ & 0.352 \\
CCTs $(\mu \mathrm{m})$ & $553 \pm 15.8$ & $552.7 \pm 11.5$ & $(533-576)$ & \\
& $(510-565)$ & $(521-572)$ & & \\
\hline
\end{tabular}

IOP, intraocular pressure; CCTs, central corneal thickness; PAD, peripheral arterial disease; DM, diabetes mellitus; $\mathrm{HT}$, hypertension

Table 2: Mean RNFL thickness in all quadrants and overall for normal and PAD groups

\begin{tabular}{lllll}
\hline & Group I & Group II & Group III & p values \\
\cline { 2 - 5 } & $($ PAD with DM/HT) $n=18$ & $($ PAD without DM/HT) $n=8$ & (Controls) $n=22$ & (Group I-III/II-III) \\
\hline TS & $135 \pm 22$ & $120 \pm 18.7$ & $123 \pm 18.2$ & $0.06 / 0.96$ \\
& $(96-171)$ & $(94-151)$ & $(93-169)$ & \\
NS & $107 \pm 16.6$ & $115 \pm 20.7$ & $137 \pm 26.7$ & $\leq 0.001 / 0.052$ \\
& $(83-143)$ & $(87-145)$ & $(96-199)$ & \\
$T$ & $69 \pm 11$ & $68.75 \pm 17.6$ & $77.5 \pm 11$ & $0.005 / 0.02$ \\
& $(52-104)$ & $(57-111)$ & $(57-98)$ & \\
$N$ & $67 \pm 14$ & $85.12 \pm 21.5$ & $63.6 \pm 16$ & $0.623 / 0.081$ \\
& $(28-90)$ & $(66-127)$ & $(4-85)$ & \\
TI & $140 \pm 19$ & $135.1 \pm 34.6$ & $135 \pm 21.2$ & $0.712 / 0.062$ \\
& $(90-166)$ & $(52-158)$ & $(85-163)$ & \\
NI & $110 \pm 21.9$ & $112.2 \pm 22.2$ & $146.4 \pm 14.14$ & $\leq 0.001 / \leq 0.001$ \\
\end{tabular}

$\mathrm{PAD}$, peripheral arterial disease; $\mathrm{DM}$, diabetes mellitus; $\mathrm{HT}$, hypertension

Table 3: Mean RNFL thickness in PAD patients

\begin{tabular}{llll}
\hline & \multicolumn{2}{c}{ Group I } & \\
\cline { 2 - 3 } & $(P A D$ with $D M / H T) n=18$ & $(P A D$ without $D M / H T) n=8$ & p values \\
\hline TS & $135 \pm 22$ & $120 \pm 18.7$ & 0.20 \\
& $(96-171)$ & $(94-151)$ & \\
NS & $107 \pm 16.6$ & $115 \pm 20.7$ & 0.54 \\
& $(83-143)$ & $(87-145)$ & \\
$T$ & $69 \pm 11$ & $68.75 \pm 17.6$ & 0.29 \\
& $(52-104)$ & $(57-111)$ & \\
$N$ & $67 \pm 14$ & $85.12 \pm 21.5$ & 0.01 \\
& $(28-90)$ & $(66-127)$ & \\
TI & $140 \pm 19$ & $135.1 \pm 34.6$ & 0.86 \\
& $(90-166)$ & $(52-158)$ & \\
NI & $110 \pm 21.9$ & $112.2 \pm 22.2$ & 0.84 \\
& $(69-129)$ & $(77-140)$ & \\
\hline
\end{tabular}

PAD, peripheral arterial disease; DM, diabetes mellitus; $\mathrm{HT}$, hypertension

Table 4: Aortic stiffness index and aortic distensibility values of the three groups

\begin{tabular}{lllll}
\hline & \multicolumn{1}{l}{ Group I } & Group II & Group III & \\
\cline { 2 - 4 } & (PAD with DM/HT) $n=18$ & $($ PAD without DM/HT) $n=8$ & (controls) $n=22$ & $p$ values \\
\hline Aortic distensibility (AD) & $0.18 \pm 0.16$ & $0.11 \pm 0.05$ & $0.5 \pm 0.2$ & $\leq 0.001$ \\
& $(0.04-0.64)$ & $(0.05-0.21)$ & $(0.31-1.08)$ & \\
Aortic stiffness B index (ASBI) & $52 \pm 37.8$ & $42 \pm 35.8$ & $14 \pm 3.97$ & $\leq 0.001$ \\
& $(11.69-156.17)$ & $(27.1-134.7)$ & $(8.07-22.99)$ & \\
\hline
\end{tabular}

PAD, peripheral arterial disease; DM, diabetes mellitus; $H T$, hypertension 
Table 5: Correlation between RNFL thickness and aortic elasticity parameters in PAD patients and the control group

\begin{tabular}{|c|c|c|c|c|c|c|}
\hline & TS & NS & $T$ & $N$ & $T I$ & $\mathrm{NI}$ \\
\hline \multicolumn{7}{|c|}{ Group I (PAD with DM/HT) } \\
\hline \multicolumn{7}{|l|}{$A D$} \\
\hline$p$ value & 0.310 & 0.874 & 0.920 & 0.355 & 0.556 & 0.648 \\
\hline$R$ value & 0.205 & -0.400 & -0.250 & 0.232 & -0.149 & -0.116 \\
\hline \multicolumn{7}{|l|}{ ASBI } \\
\hline$p$ value & 0.286 & 0.701 & 0.463 & 0.788 & 0.984 & 0.748 \\
\hline$R$ value & -0.266 & -0.91 & -0.85 & -0.68 & 0.01 & 0.082 \\
\hline \multicolumn{7}{|c|}{ Group II (PAD without DM/HT) } \\
\hline \multicolumn{7}{|c|}{$A D$} \\
\hline$p$ value & 0.568 & 0.204 & 0.670 & 0.799 & 0.365 & 0.608 \\
\hline$R$ value & -0.204 & -0.503 & 0.180 & 0.108 & -0.371 & 0.216 \\
\hline \multicolumn{7}{|l|}{ ASBI } \\
\hline$p$ value & 0.493 & 0.233 & 0.651 & 0.912 & 0.385 & 0.693 \\
\hline$R$ value & 0.268 & 0.476 & -0.190 & 0.582 & 0.357 & -0.167 \\
\hline \multicolumn{7}{|c|}{ Group III (controls) } \\
\hline \multicolumn{7}{|c|}{$A D$} \\
\hline$p$ value & 0.939 & 0.540 & 0.472 & 0.094 & 0.397 & 0.218 \\
\hline$R$ value & -0.017 & 0.148 & 0.162 & 0.366 & 0.199 & 0.274 \\
\hline \multicolumn{7}{|l|}{ ASBI } \\
\hline$p$ value & 0.231 & 0.459 & 0.343 & 0.602 & 0.877 & 0.243 \\
\hline$R$ value & 0.266 & -0.167 & -0.212 & -0.118 & -0.35 & -0.360 \\
\hline
\end{tabular}

PAD, peripheral arterial disease; DM, diabetes mellitus; $\mathrm{HT}$, hypertension

caused by HT may cause autoregulation disorders in the vascular beds, resulting in perfusion disorders in the posterior ciliary arteries and therefore the optic nerve. These conditions due to atherosclerosis result in decreased ocular blood flow and ischemic damage to the anterior optic nerve. ${ }^{29}$

Hayreh found that the RNFL visibility determined by color stereoscopic fundus photographs was significantly lower in the hypertensive and atherosclerotic monkeys than in the healthy monkey group, and localized RNFL defects were significantly higher in the patient group. ${ }^{29}$

In our study, we assessed the RNFL thickness in PAD patients (with DM or HT and without DM or HT) and age-matched healthy control subjects using the HRT that is a confocal scanning laser ophthalmoscope providing quantitative and real-time information about RNFL.

We found that the RNFL in PAD patients was significantly thinner than those of healthy subjects in superior-nasal, temporal, inferior-nasal quadrants $(p<0.05)$. Furthermore, nasal quadrant RNFL thickness in PAD patients with DM and HT is significantly thinner than that of PAD patients without DM or HT.

These results demonstrated that localized RNFL thicknesses are significantly decreased in PAD patients with DM or HT than controls. A decrease in RNFL thickness measurements in these patients may be explained by the ocular perfusion defects and optic disc head ischemia due to diabetes and HT.

Moreover, in PAD patients without HT and DM, RNFL measurements were found to be decreased especially in temporal and inferior-nasal quadrants compared with healthy controls. This result suggested that isolated $P A D$ diagnosed with $A B I$ might lead to localized RNFL loss independent of other systemic conditions like HT and DM.
Aortic distensibility ASBI is usually accepted as an indication for the early stage in atherosclerotic process. ${ }^{30}$

It is shown that $\mathrm{HT}$ and DM have a negative impact on arterial stiffness. ${ }^{30}$ We determined that PAD patients had deteriorated aortic elastic features compared with age-matched healthy subjects as expected.

Additionally, patients with PAD without HT and DM have also decreased RNFL thickness parameters. However, RNFL thickness and aortic elasticity parameters did not show any significant correlation in each group in our study.

According to literature knowledge, this study is the first to show RNFL changes in patients with isolated PAD without DM and HT diagnosed with aortic elasticity parameters ( $A D$ and $A B I)$ and also investigating the correlation between RNFL thickness and $A D$ and $A B I$ in these patients. However, these results might be attributable to the small sample size of groups as a limitation of the current study. This idea might be improved and the results of the study might be supported if patients with glaucoma underwent examination by using these aortic elasticity parameters in the future.

\section{Conclusion}

To the best of our knowledge, this is the first study showing RNFL changes in patients with isolated PAD without DM and HT diagnosed with aortic elasticity parameters ( $A D$ and $A B I)$ and also investigating the correlation between RNFL thickness and $A D$ and $A B I$ in these patients. However, these results might be attributable to the small sample size of groups as a limitation of the current study. This idea might be improved and the results of the study might be supported if patients with glaucoma underwent examination by using these aortic elasticity parameters in the future. 


\section{References}

1. Ruckley CV. Symptomatic and asymptomatic disease. In: Fowkes FGR, ed. Epidemiology of peripheral vascular disease. London: Springer; 1991. pp. 127-140.

2. Newman AB, Siscovick DS, Manolio TA, et al. Ankle arm index as a marker of atherosclerosis in the cardiovascular health study. Circulation 1993;88(3):837-845. DOI: 10.1161/01.cir.88.3.837.

3. Selvin E, Erlinger TP. Prevalence of and risk factors for peripheral arterial disease in the United States: results from the National Health and Nutrition Examination Survey, 1999-2000. Circulation 2004;110(6):738-743. DOI: 10.1161/01.CIR.0000137913.26087.F0.

4. William R, Hiatt WR. Medical treatment of peripheral arterial disease and claudication. N Engl J Med 2001;344(21):1608-1621.DOI: 10.1056/ NEJM200105243442108.

5. Davies MJ. A macro and micro view of coronary vascular insult in ischemic heart disease. Circulation 1990;82(Suppl 3):I138-II46.

6. Bots ML, Hofman A, De Jong PTVM, et al. Common carotid intimamedia thickness as an indicator of atherosclerosis at other sites of the carotid artery: the rotterdam study. Ann Epidemiol 1996;6(2):147-153. DOI: 10.1016/1047-2797(96)00001-4.

7. Witteman JCM, Grobbee DE, Valkenburg HA, et al. Cigarette smoking and the development and progression of aortic atherosclerosis. A 9-year population-based follow-up study in women. Circulation 1993;88(5 Pt 1):2156-2162. DOI: 10.1161/01.cir.88.5.2156.

8. Bots ML, Van Swieten JC, Breteler MM, et al. Cerebral white matter lesions and atherosclerosis in the Rotterdam study. Lancet 1993;341(8855):1232-1237. DOI: 10.1016/0140-6736(93) 91144-b.

9. Dhaliwal D, Mukherjee D. Peripheral arterial disease: epidemiology, natural history, diagnosis and treatment. Int Angiol 2007;16(2):36-44. DOI: 10.1055/s-0031-1278244.

10. Paraskevas KI, Kotsikoris I, Koupidis SA, et al. Ankle-brachial index: a marker of both peripheral arterial disease and systemic atherosclerosis as well as a predictor of vascular events. Angiology 2010;61(6):521-523. DOI: 10.1177/0003319710371620.

11. Sutton-Tyrrell K, Najjar SS, Boudreau RM, et al. Health $A B C$ study: elevated aortic pulse wave velocity, a marker of arterial stiffness, predicts cardiovascular events in well-functioning older adults. Circulation 2005;111(25):3384-3390. DOI: 10.1161/ CIRCULATIONAHA.104.483628.

12. Willum-Hansen T, Staessen JA, Torp-Pedersen C, et al. Prognostic value of aortic pulse wave velocity as index of arterial stiffness in the general population. Circulation 2006;113(5):664-670. DOI: 10.1161/ CIRCULATIONAHA.105.579342.

13. McEniery CM, Wilkinson IB, Avolio AP. Does arterial stiffness predict atherosclerotic coronary events? Adv Cardiol 2007;44:160-172. DOI: $10.1159 / 000096728$.

14. Malayeri AA, Natori S, Bahrami H, et al. Relation of aortic wall thickness and distensibility to cardiovascular risk factors (from the multi-ethnic study of atherosclerosis [MESA]). Am J Cardiol 2008;102(4):491-496. DOI: 10.1016/j.amjcard.2008.04.010.

15. Hayreh SS, Jonas JB. Appearance of the optic disk and retinal nerve fiber layer in atherosclerosis and arterial hypertension: an experimental study in rhesus monkeys. Am J Ophthalmol 2000;130(1):91-96. DOI: 10.1016/s0002-9394(00)00387-1.

16. Doobay AV, Anand SS. Sensitivity and specificity of the ankle bronchial index to predict future cardiovascular outcomes. a systemic review. Arterioscler Thromb Vasc Biol 2005;25(7):1463-1469. DOI: 10.1161/01. ATV.0000168911.78624.b7.

17. Shah AM, Banerjee T, Mukherjee D. Coronary, peripheral and cerebrovascular disease: a complex relationship. J Indian Med Assoc 2010;108(5):292-294.

18. Mukherjee D, Cho L. Peripheral arterial disease: considerations in risks, diagnosis, and treatment. J Natl Med Assoc 2009;101(10):999-1008. DOI: 10.1016/s0027-9684(15)31066-x.

19. Roger VL, Go AS, Lloyd-Jones DM, et al. Heart disease and stroke statistics-2012 update: a report from the American Heart Association. Circulation 2012;125(1):e2-e220. DOI: 10.1161/ CIR.0b013e318245fac5.

20. Belch JJF, Topol EJ, Agnelli G, et al. Prevention of atherothrombotic disease network: critical issues in peripheral arterial disease detection and management: a call to action. Arch Int Med 2003;163(8):884-892. DOI: 10.1001/archinte.163.8.884.

21. Lonneville $\mathrm{YH}$, Ozdek SC, Onol M, et al. The effect of blood glucose regulation on retinal nerve fiber layer thickness in diabetic patients. Ophthalmologica 2003;217(5):347-350. DOI: 10.1159/000071350.

22. Chihara E, Zhang S. Analysis of diabetic optic neuropathy with a topographic laser scanning system. Nippon Ganka Gakkai Zassi 1998;102(7):431-435.

23. Ozdek S, Lonneville $\mathrm{YH}$, Onol M, et al. Assessment of nerve fiber layer in diabetic patients with scanning laser polarimetry. Eye 2002;16(6):761-765. DOI: 10.1038/sj.eye.6700207.

24. Königsreuther KA, Jonas JB. Optic disc morphology in diabetes mellitus. Graefes Arch Clin Exp Ophthalmol 1995;233(4):200-204. DOI: $10.1007 /$ BF00183592.

25. Weinreb RN, Shakiba S, Zangwill L. Scanning laser polarimetry to measure the nerve fiber layer of normal and glaucomatous eyes. Am J Ophthalmol 1995;119(5):627-636. DOI: 10.1016/s0002-9394(14)702211.

26. Gardner TW, Antonetti DA, Barber AJ, et al. Diabetic retinopathy: more than meets the eye. Surv Ophthalmol 2002;47(Suppl):S253-S262. DOI: 10.1016/s0039-6257(02)00387-9.

27. Zhang L, Inoue M, Dong K, et al. Retrograde axonal transport of largeand medium-sized retinal ganglion cells in diabetic rat. Curr Eye Res 2000;20(2):131-136. DOI: 10.1076/0271-3683(200002)2021-DFT131.

28. Hayreh SS. Blood flow in the optic nerve head and factors that may influence it. Prog Retin Eye Res 2001;20(5):595-624. DOI: 10.1016/ s1350-9462(01)00005-2.

29. Vitarelli A, Giordano M, Germanò G, et al. Assessment of ascending aorta wall stiffness in hypertensive patients by tissue Doppler imaging and strain Doppler echocardiography. Heart 2010;96(18):1469-1474. DOI: 10.1136/hrt.2010.198358

30. Karamitsos TD, Karvounis HI, Didangellos TP, et al. Usefulness of colour tissue Doppler imaging in assessing aortic elastic properties in type 1 diabetic patients. Diabet Med 2006;23(11):1201-1206. DOI: 10.1111/j.1464-5491.2006.01974.x. 